\title{
Parámetros teórico-experimentales de una antena de cruz
}

\section{Theoretical-experimental parameters of a cross antenna}

\author{
J. Sosa-Pedroza, A. Lucas-Bravo y J. López-Bonilla**iD \\ ESIME-Zacatenco, Instituto Politécnico Nacional, México
}

\begin{abstract}
Resumen. La antena de cruz es una estructura impresa de ganancia media y polarización circular, que consiste de un conductor o cinta sobre un plano de tierra que sigue el contorno de una cruz con cuatro o más ramas y un diámetro de alrededor de 1.5 longitudes de onda desarrollada por Antoine Roederer 11. La antena se alimenta por medio de una línea coaxial y está terminada en una impedancia de carga, por lo que presenta un comportamiento de onda progresiva. Aunque en principio la antena nació para aplicaciones en comunicaciones móviles en Banda L (1500 Mhz), se presenta en este trabajo la caracterización experimental de una antena de cruz de ocho ramas que trabaja en $10 \mathrm{GHz}$, con la intención principal de servir como alimentador de un reflector parabólico para comunicaciones satelitales.
\end{abstract}

Palabras Claves. Comunicaciones satelitales, antena de Cruz, modelo de Roederer, reflector parabólico..

\begin{abstract}
The cross antenna is a medium gain, circularly polarized, printed structure consisting of a conductor or ribbon on a ground plane that follows the contour of a cross with four or more branches and a diameter of about 1.5 wavelengths developed by Antoine Roederer [1]. The antenna is fed by a coaxial line and is terminated at a load impedance, so it has a progressive wave behaviour. Although in principle the antenna was born for applications in mobile communications in L Band (1500 Mhz), it is presented in this work the experimental characterization of a cross antenna of eight branches that works in $10 \mathrm{GHz}$, with the main intention to serve as a feeder of a parabolic reflector for satellite communications.
\end{abstract}

Keywords. Satellite communications, Roederer's model, parabolic reflector, cross antenna.

Como citar. J. Sosa-Pedroza, A. Lucas-Bravo y J. López-Bonilla, "Parámetros teórico-experimentales de una antena de cruz", Jou. Cie. Ing., vol. 11, no. 1, pp. 65-71, 2019. doi: 10.46571/JCl.2019.1.7

\section{Introducción}

La antena de cruz pertenece a la familia de radiadores de onda progresiva, formada por una microlínea con un plano de tierra, la alimentación en un extremo y una carga en el otro; posee polarización circular derecha o izquierda dependiendo de la posición de la alimentación y de la carga; puede ser usada en forma independiente o como radiador primario de reflectores parabólicos con relaciones grandes entre longitud focal y diámetro. La ventaja de las antenas de cruz sobre otras de ganancia media $(12-15 \mathrm{dBi})$ son sus dimensiones longitudinales, mucho menores que las antenas de hélice, corneta, etc.

*e-mail: jlopezb@ipn.mx 
En comparación con otras antenas impresas, como parches o dipolos cruzados, la antena de cruz tiene la ventaja de reducir los problemas de acoplamiento y por tanto de alimentación, lo que se demuestra claramente en los resultados obtenidos en este trabajo.

\section{La Estructura}

La geometría de la antena se muestra en la figura 1, una cinta en forma de cruz, impresa sobre un plano de tierra a una distancia de una fracción de la longitud de onda de trabajo. La línea se alimenta en un extremo, por medio de un cable coaxial y se termina en el otro extremo por una carga. La longitud de las ramas de la cruz se seleccionan para que haya un defasamiento en la corriente, entre una y la contigua, de $2 \pi+\frac{2 \pi}{N}$, donde $N$ es el número de ramas. Como el campo eléctrico radiado por cada rama gira $\frac{2 \pi}{N}$, el campo total tendrá polarización circular, si no existe atenuación a lo largo de la cinta; en la práctica debe buscarse que la atenuación sea lo menor posible.

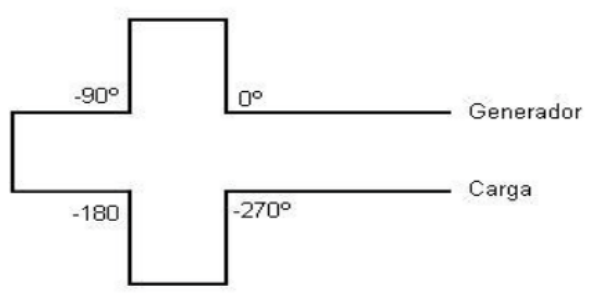

Figura 1: Antena de Cruz.

Para una antena de cuatro ramas, los brazos largos tienen una longitud de $\lambda e / 2$ y los brazos $\lambda e / 4$ donde $\lambda e$ representa la longitud de onda efectiva, determinada por el dieléctrico de la microcinta. Dos pares sucesivos de ramas largas radían un campo paralelo al conductor, con amplitudes decreciendo hacia el final de la línea. El brazo corto introduce un defasamiento de $90^{\circ}$ entre los campos radiados por pares sucesivos y además contribuye con el campo en la dirección perpendicular.

Como otras antenas de onda progresiva, la corriente decae exponencialmente a lo largo de la línea, aunque en este caso con ondulaciones debidas a reflexiones en los dobleces. La potencia absorbida o reflejada al final de la línea se controla por medio de la impedancia de carga y puede limitarse a un pequeño porcentaje de la potencia total ajustando también la altura de la línea sobre el plano de tierra (típicamente $\lambda e / 20$ a $\lambda e / 4$ ). Por otro lado, la longitud de la línea puede optimizarse cambiando el número de brazos y vueltas de la antena (típicamente entre 5 y 15 $\lambda e$ ), de lo cual también depende el ancho de banda de la antena, que oscila alrededor del $5 \%$. Las ondas reflejadas por dobleces sucesivos tienden a cancelarse entre sí por lo que su influencia es pequeña en el comportamiento total del radiador [2, 3].

El ancho de banda de la antena depende del número de brazos y vueltas, pero como otras antenas de onda progresiva, el ancho de banda es alrededor de $5 \%$. Un elemento importante en la antena es la impedancia de carga, aunque la forma de la antena hace que la corriente disminuya al viajar a través de ella, dejando muy poca energía en la terminal de carga, la impedancia al final debe prácticamente eliminarla, por otro lado se usa para ajustar la relación axial de la polarización circular, igualmente Roederer menciona que el ajuste de la impedancia de carga puede reducir la polarización cruzada.

En este trabajo se usó como impedancia de carga la misma impedancia medida a la entrada de la antena, considerando la simetría de la estructura, el valor de la impedancia de entrada se obtuvo haciendo mediciones de impedancia en circuito abierto y en circuito corto y usando la conocida expresión [2]: 


$$
Z_{0}=\sqrt{Z_{c c} Z_{c a}} \ldots \ldots \ldots 1
$$

El trabajo experimental se dividió en dos etapas, la primera fue la caracterización de la impedancia de entrada y la segunda la medición de acoplamiento de impedancia, de ganancia y de patrón de radiación, en dos direcciones para calcular la relación axial, construyendo antenas para dos dieléctricos: Duroid y aire, con diferente separación con el plano de tierra.

Para la caracterización de impedancia y reflexión se usó un analizador de redes HP8510; la medición de patrón de radiación y ganancia se hizo en un generador de RF Wiltron 68147B y un analizador de espectro Advantest R3272,. Como referencia se usó una antena de corneta de $12 \mathrm{dBi}$. La potencia de alimentación fue de $10 \mathrm{dBmW}$.

Las antenas construidas son simétricas de una vuelta, con ocho brazos, terminadas en su impedancia característica. La geometría de la antena se muestra en la figura 2 , trabaja a una frecuencia de $10 \mathrm{GHz}$, con una longitud de onda de $3 \mathrm{~cm}$. para el aire y 1.96 para el Duroid $\left(\varepsilon_{r}\right.$ $=2.33$ ). La tabla muestra las características geométricas, donde $\lambda e$ representa la longitud de onda efectiva.

\begin{tabular}{|c|c|}
\hline Longitud del Brazo & $1 \lambda e$ \\
\hline Ancho del Brazo & $0.25 \lambda e$ \\
\hline Diámetro de la Cruz & $2 \lambda e$ \\
\hline Diámetro Conductor & $0.01 \lambda e$ \\
\hline
\end{tabular}

Tabla 1: Características Geométricas

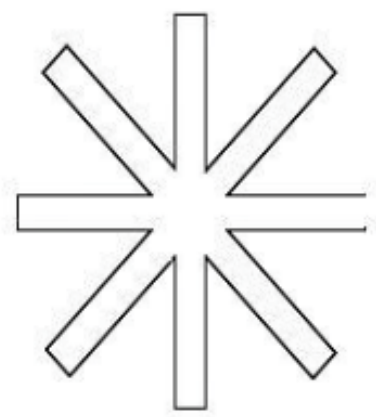

Figura 2: Esquema de la antena.

Para el caso del Duroid se construyó una antena con un espesor de dieléctrico de $3.6 \mathrm{~mm}$ y para el segundo se construyó una antena de alambre de cobre, montada sobre un plano de tierra, esta construcción dio mayor flexibilidad para determinar el efecto de la altura de la antena sobre el plano de tierra, tanto en el patrón de radiación como en el acoplamiento. Para darle estabilidad mecánica, mantener su posición y cambiar la altura, fue soportada por pequeños fragmentos de teflón localizados en algunos puntos de la estructura.

\section{Impedancia de carga}

La simetría de la antena nos lleva a suponer que tiene la misma impedancia en ambos extremos, por lo que el primer paso del experimento fue su determinación. Se midió la impedancia en corto circuito y en circuito abierto y a partir de esos valores se calculó la impedancia de entrada usando la ecuación 1, la tabla 2 muestra las mediciones y el cálculo en varias frecuencias. 


\begin{tabular}{|l|l|l|l|}
\hline $\mathbf{f}(\mathbf{G H z})$ & $Z_{c c}$ medida & $Z_{c a}$ medida & $Z_{0}=\left(Z_{c c}^{*} Z_{c a}\right)^{\frac{1}{2}}$ \\
\hline 8 & $47.8+\mathrm{j} 15$ & $45+\mathrm{j} 17$ & $46.4+\mathrm{j} 16$ \\
\hline 8.5 & $47.3+\mathrm{j} 28$ & $54.7+\mathrm{j} 13.4$ & $51.5+\mathrm{j} 21$ \\
\hline 9 & $65+\mathrm{j} 4.5$ & $56.6-\mathrm{j} 13.5$ & $61.4-\mathrm{j} 5.1$ \\
\hline 9.5 & $27.4+\mathrm{j} 12$ & $28.6+\mathrm{j} 14.4$ & $28+\mathrm{j} 13.2$ \\
\hline 10 & $59+\mathrm{j} 0.3$ & $72.8-\mathrm{j} 11.2$ & $65.7-\mathrm{j} 4.8$ \\
\hline 10.5 & $54.8+\mathrm{j} 16.3$ & $48.7-\mathrm{j} 0.2$ & $52.2+\mathrm{j} 7.5$ \\
\hline 11 & $84-\mathrm{j} 1.3$ & $72.3+\mathrm{j} 13$ & $78.3+\mathrm{j} 6.4$ \\
\hline 11.5 & $57.6-\mathrm{j} 23.8$ & $62.6-\mathrm{j} 22$ & $60-\mathrm{j} 23$ \\
\hline 12 & $43.8-\mathrm{j} 18$ & $46.2-\mathrm{j} 16.7$ & $45-\mathrm{j} 17.4$ \\
\hline
\end{tabular}

Tabla 2: Medición y cálculo de impedancia

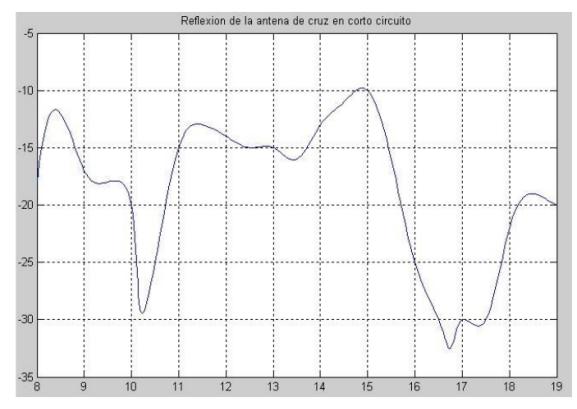

Figura 3: Reflexión en Corto Circuito.

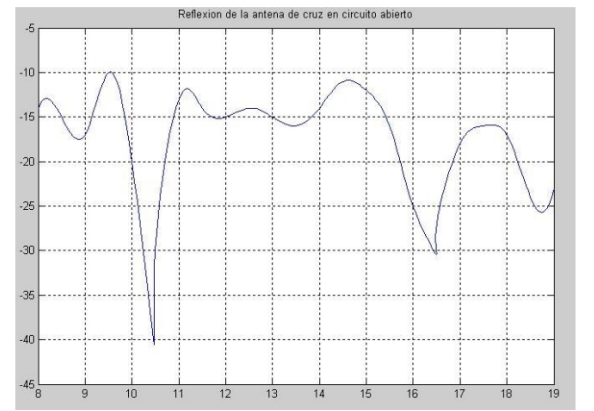

Figura 4: Reflexión en Circuito Abierto.

Se presentan en las figuras 3, 4 y 5 gráficas del coeficiente de reflexión para una antena en corto circuito, circuito abierto y con impedancia de carga de $50 \Omega$. En la 6 se muestra la impedancia de la entena con carga de $50 \Omega$.

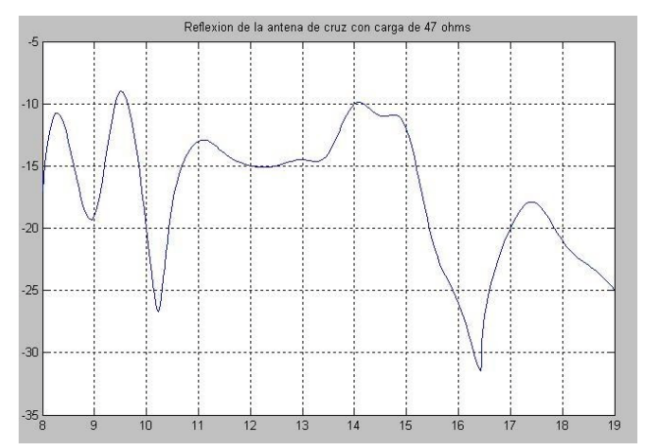

Figura 5: Reflexión en carga de $50 \Omega$.

Es interesante observar en estas gráficas las características de resonancia en 10.2 y $16.4 \mathrm{GHz}$, que se mantienen casi sin variación con las tres cargas diferentes. El resultado es una muestra de la presencia de la onda progresiva en la frecuencia de trabajo y de lo fácil que es obtener ese comportamiento con la antena de cruz, se tienen resultados similares invirtiendo las terminales de alimentación y carga aunque no se presentan aquí por ahorro de espacio. 


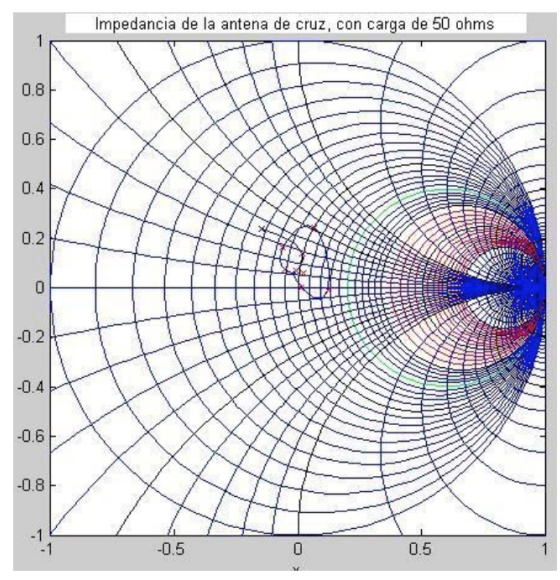

Figura 6: Impedancia con carga de $50 \Omega$.

\section{Patrón de radiación}

Las características de radiación de la antena dependen de su separación del plano de tierra, por lo que el estudio se hizo para 4 antenas, 1 de Duroid y 3 de Dieléctrico aire.

Las antenas de dieléctrico aire se construyeron de alambre de cobre 30 AWG, lo que representa una relación de $0.011 \lambda$. Las antenas se montaron sobre un plano de tierra y se soportaron con pequeños pedazos de teflón en lugares estratégicos para mantener la posición y una separación uniforme con el plano conductor. Se construyeron tres antenas con separaciones de 2, 4 y $8 \mathrm{~mm}$. Se midieron los campos para el plano de $\varphi=0^{\circ}$ y a $\varphi=90^{\circ}$, para poder determinar la relación

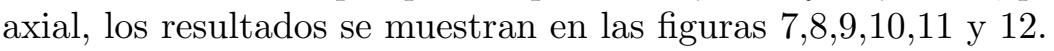

La antena de Duroid se grabó sobre un material de doble placa conductora, una de ellas usada como plano de tierra y el otro para grabar la antena. La permitividad relativa del Duroid es de 2.33, por lo que la longitud de onda a $10 \mathrm{GHz}$ es de $1.965 \mathrm{~cm}$., el ancho del dieléctrico es de $3.6 \mathrm{~mm}$., los resultados para $\varphi=0^{\circ}$ y $\varphi=90^{\circ}$ se muestran en la figuras 13 y 14 . Las ganancias obtenidas a partir de la antena patrón de corneta y la relación axial, calculada de la comparación de los valores máximos de ganancia de los patrones ortogonales, se muestran en la tabla 3 , en la dirección de máxima radiación:

\begin{tabular}{|c|c|c|}
\hline ANTENA & G dB & R.A dB \\
\hline $2 \mathrm{~mm}$. & 13.1 & 2.0 \\
\hline $4 \mathrm{~mm}$. & 11.8 & 1.2 \\
\hline $8 \mathrm{~mm}$. & 12.0 & 1.3 \\
\hline Duroid & 11.0 & 2.0 \\
\hline
\end{tabular}

Tabla 3: Ganancias y relación axial. 


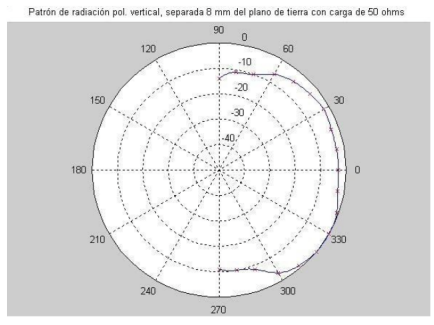

Figura 7: Diel aire, separación $8 \mathrm{~mm}$, $\varphi=0^{\circ}$.

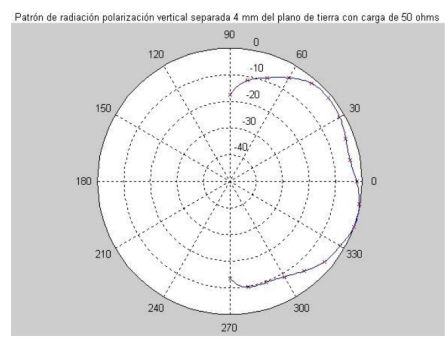

Figura 9: Diel aire, separación $4 \mathrm{~mm}$, $\varphi=0^{\circ}$.

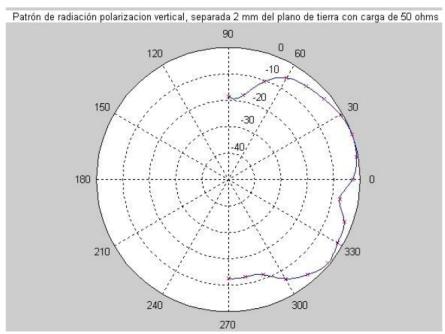

Figura 11: Diel. aire, separación 2 mm, $\varphi=0^{\circ}$.

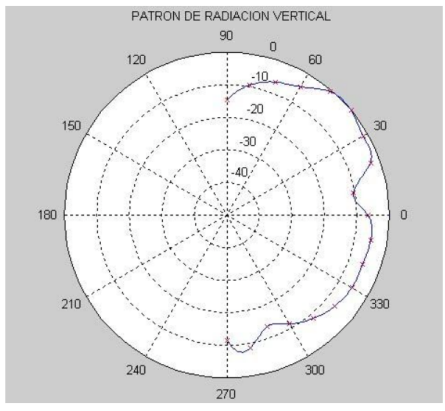

Figura 13: Diel. Duroid separación 3.6 $\mathrm{mm}, \varphi=0^{\circ}$.

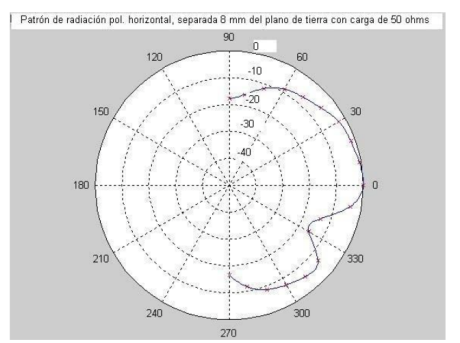

Figura 8: Diel aire, separación $8 \mathrm{~mm}$, $\varphi=90^{\circ}$.

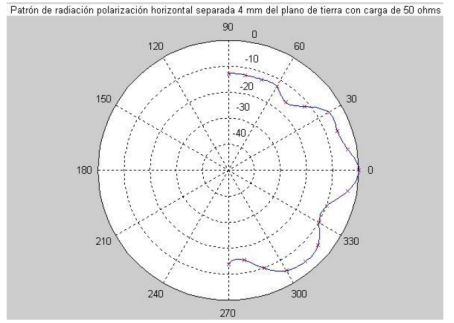

Figura 10: Diel. aire, separación $4 \mathrm{~mm}$, $\varphi=90^{\circ}$.

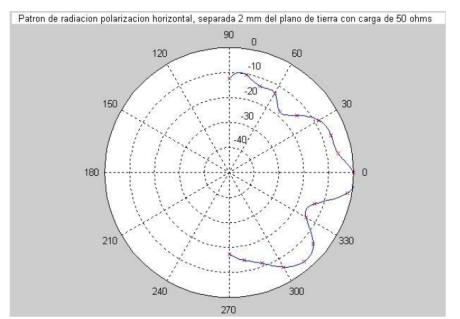

Figura 12: Diel. aire, separación $2 \mathrm{~mm}$, $\varphi=90^{\circ}$.

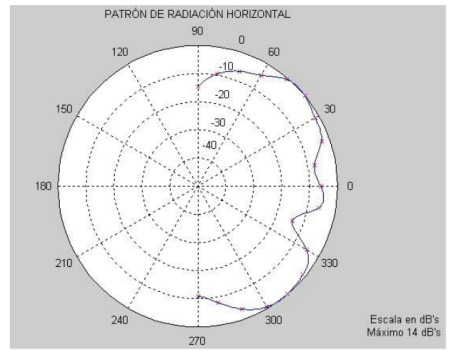

Figura 14: Diel. Duroid separación 3.6 $\mathrm{mm}, \varphi=90^{\circ}$.

\section{Conclusiones}

Se han presentado resultados experimentales obtenidos en antenas de cruz con diferentes características estructurales, la facilidad en la construcción y sobre todo la facilidad con que se obtienen características estables y repetibles en condiciones extremas, como la impedancia 
de entrada con cargas tan dispares; en la misma forma resultados de ganancia, comparables con antenas de corneta, como la usada de referencia, la hacen ideal para aplicaciones en comunicaciones a frecuencias de microondas, ya sea usada aisladamente o como radiadores primarios de reflectores parabólicos.

Los resultados de reflexión muestran que la antena resuena a la frecuencia de diseño, sin mucho problema, para diferentes impedancias de carga, lo que nos lleva a concluir que existe una onda de corriente progresiva, es decir la onda reflejada en el extremo final es casi nula, como corresponde a este tipo de antena. Las mediciones con una carga de 50 , que se presentan en carta de Smith, son comparables con los resultados calculados y muestran también que la impedancia en un ancho de banda considerable ( 8 a $20 \mathrm{GHz}$ ), se mantiene cerca del centro de la carta. Por otro lado, la ganancia es muy similar a la de la corneta con las ventajas que se tienen en relación al peso y las dimensiones, además de una relación axial muy aceptable en el máximo de radiación.

El análisis de los patrones de radiación, muestra también estabilidad, la separación con el plano de tierra afecta poco la distribución de campo electromagnético en el plano $\varphi=0^{\circ}$, pero la diferencia es muy marcada en el plano ortogonal, en este caso los patrones se hacen más directivos, con una mejor distribución para una separación de 4mm, sin embargo, en los tres casos existe una deformación entre $-30^{\circ}$ y $-60^{\circ}$. El reporte de Roederer muestra la misma deformación, aunque no tan marcada, sin embargo, él construye a $1.5 \mathrm{GHz}$, mientras que a la frecuencia de nuestro trabajo, la longitud de onda es casi 7 veces menor y las dimensiones de la resistencia o las características del conector podrían ser la diferencia. El trabajo continúa sobre todo para reducir el efecto del conector, para mejorar la relación axial y para el efecto de la segunda frecuencia de resonancia.

\section{Referencias}

[1] A. G. Roereder, "The Cross Antenna: a New Low Profile Circularly Polarized Radiator", IEEE Transactions on Antennas and Propagation, vol. 38, no. 5, 1990.

[2] J. Sosa-Pedroza, L. Ortega Lara, Lineas de Transmisión y Guías de Onda, México: Ed. Limusa, 1990.

[3] A. Lucas-Bravo, J. Sosa-Pedroza, J. López-Bonilla, "Cross antenna: An experimental and numerical analysis", Apeiron, vol. 13, no. 2, pp. 274-287, 2006.
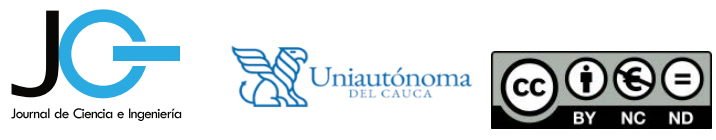